\title{
View and Visits of Articles on Neglected Tropical Diseases in the Malaysian Medical Gazette Website
}

\author{
Aneesa Abdul Rashid ${ }^{1}$,Hidayatul Radziah Ismawi \\ ${ }^{1}$ Department of Family Medicine, Faculty of Medicine and Health Sciences, Universiti Putra Malaysia, \\ Serdang, Malaysia; ${ }^{2}$ Department of Basic Medical Sciences, Kulliyyah of Medicine, International \\ Islamic University Malaysia
}

\begin{abstract}
Introduction: The Malaysian Medical Gazette (MMG) is an online source of credible, informative and easily understandable articles for public consumption written by real doctors and healthcare professionals. Launched online at www.mmgazette.com by the Director General of Health, Malaysia. Among the articles published are those on Neglected Tropical Diseases (NTD), namely dengue, melioidosis, rabies and leprosy. These diseases have caused signific ant morbidity and mortality in Malaysia; hence they are aimed to educate the public.
\end{abstract}

Objective: We aim to determine the number of views and entries of articles on NTDs in the MMG from July 2018 until June 2019.

Methods: This is a cross-sectional study looking into data analytics of the MMG website. The number of unique views and entries (the first page that is directly linked to) on NTD articles are identified and compared to the total number of views and visits of the entire website.

Results: There are a total of 4,739,949 of total views on the MMG website. Out of that a total of $40,772(0.86 \%)$ are views on NTD articles. The MMG website received 1,436,969 total visits from July 2018 to June 2019, with $13,850(0.96 \%)$ unique to NTD articles. A total of 35,913 were views on dengue articles whereby $11,511(32.1 \%)$ were the unique visits by users. Views in Bahasa were more popular $(27,487)$ compared to English $(8,426)$. Other NTD views were quite modest for Rabies $(2,170)$, Leprosy $(2,076)$ and Melioidosis $(613)$ in the past year.

Conclusion: We found users of MMG to have a high number of views, with more than 40,000 views on NTD articles. Users preferred articles on dengue, and in Bahasa Malaysia as opposed to the English language. More studies should investigate the preference of online health site user in Malaysia to reach and educate Malaysian users.

Keywords: online health; neglected; tropical diseases; dengue; digital health 